\title{
A clustering- and probability-based approach for time-multiplexed FPGA partitioning
}

\author{
Guang-Ming $\mathrm{Wu}^{\mathrm{a}, *}$, Mango Chia-Tso Chao ${ }^{\mathrm{b}}$, Yao-Wen Chang ${ }^{\mathrm{c}}$ \\ ${ }^{a}$ Department of Information Management, Nan-Hua University, 32 Chung Keng, Dalin, Chiayi, Taiwan \\ ${ }^{\mathrm{b}}$ Computer and Information Science, National Chiao Tung University, Hsinchu 30010, Taiwan \\ ${ }^{\mathrm{c}}$ Department of Electrical Engineering, National Taiwan University, Taipei, Taiwan
}

Received 14 February 2003; received in revised form 25 May 2004; accepted 3 June 2004

\begin{abstract}
Improving logic density by time-sharing, time-multiplexed FPGAs (TMFPGAs) have become an important research topic for reconfigurable computing. Due to the precedence and capacity constraints in TMFPGAs, the clustering and partitioning problems for TMFPGAs are different from the traditional ones. In this paper, we propose a two-phase hierarchical approach to solve the partitioning problem for TMFPGAs. With the precedence and capacity considerations for both phases, the first phase clusters nodes to reduce the problem size, and the second phase applies a probability-based iterative-improvement approach to minimize cut cost. Experimental results based on the Xilinx TMFPGA architecture show that our algorithm significantly outperforms previous works.
\end{abstract}

(C) 2004 Elsevier B.V. All rights reserved.

Keywords: Layout; Physical_design; Partitioning

\section{Introduction}

Improving logic density by time-sharing, time-multiplexed FPGAs (TMFPGAs) have become an important research topic for reconfigurable computing. In TMFPGAs, a virtual large design is

\footnotetext{
${ }^{*}$ Corresponding author. Tel.: $+88652721001 \times 201$; fax: +88652427136 .

E-mail addresses: gmwu@mail.nhu.edu.tw (G.-M. Wu), gis87530@cis.nctu.edu.tw (M.C.-T. Chao), ywchang@ cc.ee.ntu.edu.tw (Y.-W. Chang).
} 


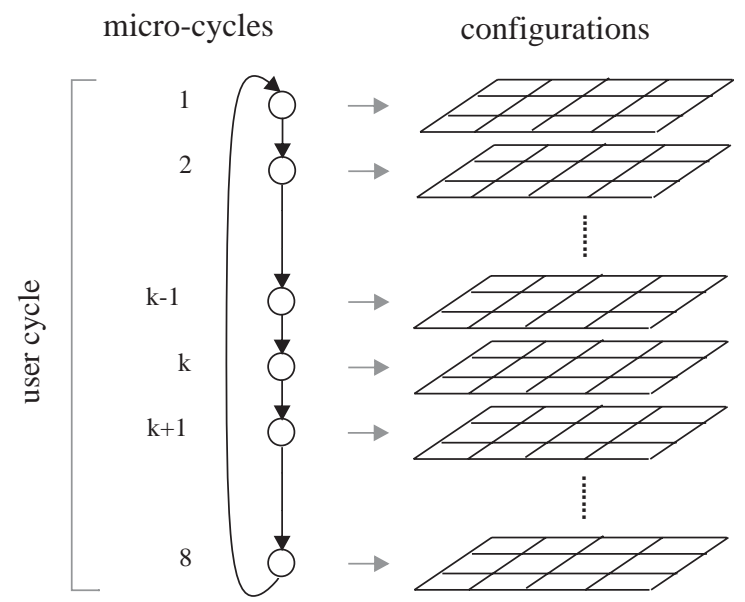

Fig. 1. The Xilinx time-multiplexed FPGA configuration model.

partitioned into multiple stages (or partitions) to share the same smaller physical device than that occupied by traditional FPGAs. Several different architectures have been proposed, such as the Xilinx model [1], Dharma [2], etc. All these models allow dynamic reuse of logic blocks and wire segments by using more than one on-chip SRAM bit to control them. The configurations of logic blocks and wire segments can be changed by reading different SRAM bits.

Fig. 1 shows the Xilinx TMFPGA configuration model [1]. The TMFPGA emulates a single circuit design in the sequencing of multiple configurations. In each micro-cycle, the TMFPGA reads in the circuit information from a corresponding configuration SRAM, and then the configurable logic blocks (CLBs) in the TMFPGA are reused to evaluate logic. A user cycle is a cycle passing through all micro-cycles. Each CLB contains micro registers to hold the CLB result. Micro registers hold the intermediate values of combinational logic for later micro-cycles in the same user cycle and reserve the status of flip-flops for the next user cycle. In Xilinx TMFPGAs, there are eight micro-cycles in a user cycle. A new configuration is loaded into active configuration memory after all CLB results in the last micro-cycle have been saved.

The objective of the TMFPGA partitioning problem is to minimize the interconnection (the number of micro registers required) between micro-cycles. Unlike a traditional FPGA, the execution order of nodes in a TMFPGA must follow their precedence constraints. For example, a node must be executed no later than all of its outputs in a combinational circuit. It implies that a cut in a TMFPGA partitioning should be a uni-directional cut. For the TMFPGA partitioning problem, several heuristics such as list scheduling $[3,4]$ and network-flow-based approach [5-7] on different architectures were proposed. The network-flow-based approach first finds a min cut. If the min cut is not at the balanced point, it will randomly move nodes to meet the balance constraint. Thus the optimality may deviate away after nodes are adjusted. In this paper, we propose a two-phase approach, the CPAT method (Clustering and Probability-based Algorithm for TMFPGA), to solve the TMFPGA partitioning problem. The first phase reduces the problem size using a clustering method; the second phase minimizes the interconnection by a probabilitybased iterative-improvement [8,9] method. For the first phase, we extend the method used in [10] 
which is effective in clustering traditional circuits, but may generate a cluster of size exceeding the capacity of a stage in the TMFPGA partitioning. Our solution to the capacity overflow problem is based on a rooted-tree subset-sum formulation; we prove that the rooted-tree subset-sum problem is NP-complete and present an exact exponential-time and a fully polynomial-time approximation algorithms [11] for the problem. For the second phase, the probability-based method incorporates the precedence constraints into the 2nd-order probability estimation [12]. Thus, the probabilitybased method finds the potentially maximum gain among movable nodes. Our method, thus, can globally monitor the changes and can avoid the drawback of the network-flow-based approach. Experimental results, based on the Xilinx TMFPGA architecture [1] with eight micro-cycles (stages), show that our algorithm reduces the maximum numbers of micro registers required than previous works.

\section{Problem formulation}

We follow the formulation and notation used in [5]. A circuit in a TMFPGA can be represented by a directed hypergraph $G(V, N)$, where $V$ is the set of nodes and $N$ is the set of nets in the circuit. There are two types of nodes in $V$ : combinational nodes ( $C$-nodes) and flip-flop nodes (FF-nodes). Each node $v \in V$ has a weight $w(v)$. The weight of a set $U(U \subseteq V), W(U)$, is given by $\left.\sum_{v \in U^{w}} w\right)$. For a net $n=\left\{v_{1}, v_{2}, \ldots, v_{p}\right\}$ with $p$ nodes, let $v_{1}$ be the fan-out node whose output signal is the input signal to $v_{j} \in n(2 \leqslant j \leqslant p)$, and let $v_{j} \in n(2 \leqslant j \leqslant p)$ be the fan-in node whose input signal is the output signal from $v_{1}$.

To fit into a TMFPGA, a circuit is partitioned into $k$ stages, such that the logic blocks and wire segments in different stages can share the same physical TMFPGA device. These $k$ stages form one user cycle, and one user cycle should produce the same results on the outputs as would be seen by a non-time-multiplexed device. In order to ensure the correct results produced in a user cycle, every nodes must be evaluated in a proper order. According to the Xilinx architecture [1], the following three precedence constraints must be satisfied:

1. Each combinational node (C-node) must be scheduled in a stage no later than all its output nodes.

2. Each FF-node must be scheduled in a stage no earlier than all its output nodes. This rule guarantees that all the nodes that use the value of the flip-flop use the same value: the value of flip-flop from the previous user cycle.

The above constraints define a partial temporal ordering on the nodes in the circuit. Let Pre $(v)$ be the precedence of a node $v$. For two nodes $u$ and $v$, let $\operatorname{Pre}(u) \operatorname{Pr} e(v)$ denote that node $u$ must be scheduled no later than node $v$. In other words, for a net $n=\left\{v_{1}, v_{2}, \ldots, v_{p}\right\}$, where $v_{1}$ is the fanout node and $v_{j}, 2 \leqslant j \leqslant p$, is the fan-in node.

- if $v_{1}$ is a C-node, then $\operatorname{Pre}\left(v_{1}\right) \operatorname{Pre}\left(v_{j}\right)$ for $2 \leqslant j \leqslant p$;

- if $v_{1}$ is an FF-node, then $\operatorname{Pre}\left(v_{j}\right) \operatorname{Pre}\left(v_{1}\right)$ for $2 \leqslant j \leqslant p$.

By the two constraints, we can decide the directions of nets in the graph and classify nets into two types: a net is $C$-type if its $v_{1}$ is a $\mathrm{C}$-node, and a net is FF-type if its $v_{1}$ is an FF-node, as shown in Fig. 2. 

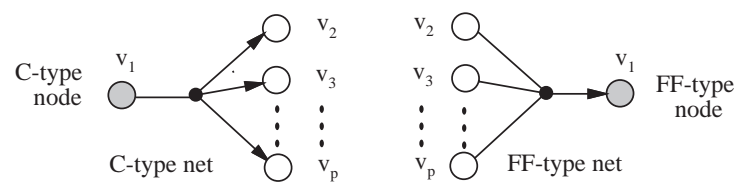

Fig. 2. Precedence constraints. Shaded nodes and white nodes represent the fan-out nodes and fan-in nodes, respectively.

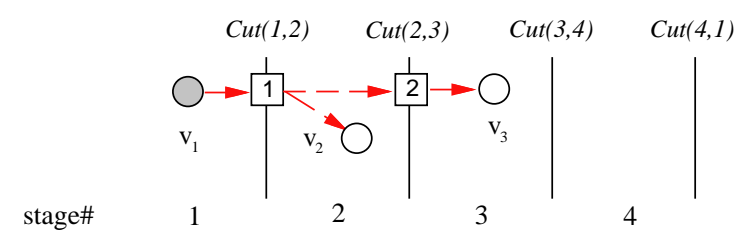

(a)

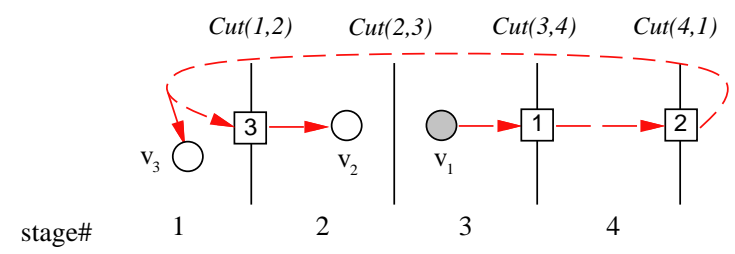

(b)

Fig. 3. (a) Two micro registers, indicated by $\square$, used in a C-type net $\left\{v_{1}, v_{2}, v_{3}\right\}$, (b) Three micro registers used in an FFtype net $\left\{v_{1}, v_{2}, v_{3}\right\}$.

In TMFPGAs, micro registers are required between stages to store the data of nodes for use in later micro-cycles. Let $C u t(a, b)$ be the set of micro registers between stage $a$ and stage $b$. A $k$-stage TMFPGA contains $k$ cuts, $\operatorname{Cut}(1,2), \operatorname{Cut}(2,3), \ldots, \operatorname{Cut}(k-1, k)$, and $\operatorname{Cut}(k, 1)$. For a C-type net, the data of its fan-out node must be held until the last stage containing a fan-in node of the net. For an FF-type net, the data of its fan-out node must be held not only in the rest stages of the current user cycle but also from the first stage to the last stage of all its fan-in nodes in the next user cycle. For a net $n=\left\{v_{1}, v_{2}, \ldots, v_{p}\right\}$, let $s(v)=j$ if $v$ belongs to the stage $j, \alpha(n)$ denote the number of micro registers used in net $n$, and $k$ denote the number of stages. $\alpha(n)$ is given as follows:

- $\alpha(n)=\max \left\{s\left(v_{j}\right) \mid 2 \leqslant j \leqslant p\right\}-s\left(v_{1}\right)$, if net $n$ is C-type.

- $\alpha(n)=k-s\left(v_{1}\right)+\max \left\{s\left(v_{j}\right) \mid 2 \leqslant j \leqslant p\right\}$, if net $n$ is FF-type.

Fig. 3 shows the registers needed in a net for a 4-stage TMFPGA. In Fig. 3(a), the data of a Ctype fan-out node is held from stage 1, the stage of the fan-out node, to stage 3 , the last stage of the fan-in nodes. It uses two micro registers, one for $\operatorname{Cut}(1,2)$ and $\operatorname{Cut}(2,3)$ each. In Fig. 3(b), the data of an FF-type fan-out node is held from stage 3, the stage of fan-out node, to stage 4, then back to stage 1 of next user cycle and finally to stage 2 , the last stage of fan-in nodes. It uses three registers, one for $\operatorname{Cut}(3,4), \operatorname{Cut}(4,1)$, and $\operatorname{Cut}(1,2)$ each. 
The $k$-stage TMFPGA partitioning problem is to partition a circuit $G(V, N)$ into $k$ nonoverlapping subsets $V_{1}, V_{2}, \ldots, V_{k}$, such that the maximum interconnection (the number of micro registers) between each two adjacent stages is minimized, and the following properties are satisfied:

(1) $\bigcup_{i=1}^{k} V_{i}=V$.

(2) Precedence constraint: Let $s(v)=j$ if $v \in V_{j}$. For each two nodes $u$ and $v$, if $\operatorname{Pre}(u) \operatorname{Pre}(v)$, then $s(u) \leqslant s(v)$.

(3) Balance constraint: For each subset $V_{i}, W\left(V_{i}\right)$ is bounded by a factor $r$ as follows:

$$
\frac{W(V)}{k}(1-r) \leqslant W\left(V_{i}\right) \leqslant \frac{W(V)}{k}(1+r), 0 \leqslant r \leqslant 1 .
$$

(4) Timing constraint: Let $D$ be the length of the longest path in a circuit. The length of the longest path in each stage is upper bounded by $\lceil D / k\rceil$.

\section{The two-phase CPAT algorithm}

The $k$-stage TMFPGA partitioning problem can be handled by repeatedly solving $k-1$ TMFPGA bipartitioning problems. We shall focus our discussions on the approach for solving the TMFPGA bipartitioning problem. Our solution to this problem is based on a two-phase hierarchical approach: clustering followed by a probability-based iterative-improvement formulation.

\subsection{Phase I: the clustering algorithm}

An effective clustering algorithm can greatly improve the quality of the precedence-constrained partitioning results and speed up the later partitioning algorithm by reducing the problem size. The maximum fanout free subgraph (MFFS) algorithm is effective in clustering traditional circuits [10]. MFFS is a signal flow-based clustering algorithm that considers simultaneous movement of logically dependent nodes during the node moves. However, MFFS may generate a cluster of size larger than the capacity of a stage in the TMFPGA partitioning. To consider the capacity constraint, we propose a clustering method based on the MFFS, which can control the size of a cluster. The definitions of FFS and MFFS are described as follows. For a given node $v$ in a circuit,

- $F F S_{v}=\{u \mid$ every path from u to some primary output passes through $v$ in the circuit $\}$.

- $\mathrm{MFFS}_{v}=\left\{u \mid\right.$ for all $\left.F F S_{v}, u \in F F S_{v}\right\}$.

A circuit can be represented in the TMFPGA by a directed graph. For a given circuit $C_{i}$ and a node $v$, an MFFS cluster rooted at $v$ can be obtained by using the following procedure:

- Convert $C_{i}$ to a directed graph, $G(V, N)$, where $V$ is a set of nodes which corresponds to $C_{i}$, and $N$ is a set of directed edges. A directed edge $(i, j)$ exists if node $j$ is a fan-in of node $i$. 


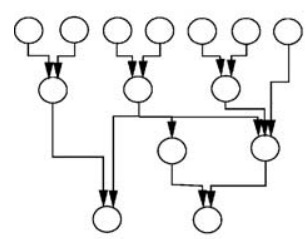

(a)

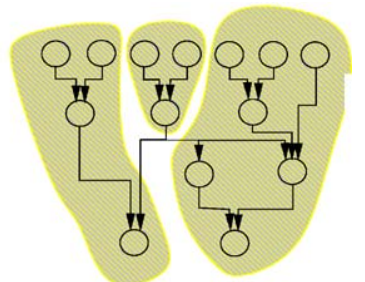

(b)

Fig. 4. (a) The original circuit, (b) Clustering by MFFS clustering.

- Cut all the fan-out edges of the root node $v$; search all other nodes in graph $G(V, N)$ starting from the primary outputs of the $C_{i}$. The nodes in $G(V, N)$ that were not traversed belong to the $M F F S_{v}$.

The MFFS construction algorithm described above is used to obtain one MFFS cluster. To cluster the entire circuit, we need to apply the MFFS construction algorithm repeatedly. The MFFS clustering algorithm works as follows: For a given circuit $C_{i}$, let Roots $=$ all primary outputs in $C_{i}$. Then, extract a node $v \in$ Roots and use the MFFS construction algorithm to construct $M F F S_{v}$. This process is repeated until Roots is empty. Then remove all currently constructed MFFS clusters from $C_{i}$, resulting in a reduced circuit $C_{i}^{\prime}$ whose primary outputs are input nodes to the removed MFFS clusters. Repeat the same procedure for the new circuit $C_{i}^{\prime}$ recursively until all nodes in $C_{i}$ are grouped into MFFS clusters. For example, the circuit depicted in Fig. 4(a) can be clustered into three clusters (see Fig. 4(b)).

We present in the following two algorithms to handle a cluster of size exceeding the capacity of a stage in the TMFPGA partitioning. Our method decomposes a cluster $C_{i}$ (rooted at $v$ ) according to the two cases: (1) $C_{i}$ is a rooted tree, and (2) $C_{i}$ is an acyclic graph. Our target is to partition $C_{i}$ into two balanced sets with the minimal cut size.

We first consider the case where a circuit $C_{i}$ is a rooted tree. Let $T_{v_{i}}$ denote the subtree rooted at $v_{i}$, where $v_{i} \in C_{i}$. For nodes $v_{1}, v_{2}, \ldots$, and $v_{d}$ in respective $T_{v_{1}}, T_{v_{2}}, \ldots$, and $T_{v_{d}}$, let $\kappa\left(v_{1}, v_{2}, \ldots, v_{d}\right)$ denote the total weights of nodes in $T_{v_{1}}, T_{v_{2}}, \ldots$, and $T_{v_{d}}$. We define an element $x=\left(\kappa\left(v_{1}, v_{2}, \ldots, v_{d}\right), T_{v_{1}}, T_{v_{2}}, \ldots, T_{v_{d}}\right)$, where $v_{1}, v_{2}, \ldots, v_{d}$ represent the respective roots of disjoint subtrees $T_{v_{1}}, T_{v_{2}}, \ldots, T_{v_{d}}$. For an element $x=\left(\kappa\left(v_{1}, v_{2}, \ldots, v_{d}\right), T_{v_{1}}, T_{v_{2}}, \ldots, T_{v_{d}}\right)$, let $|x|=d$ and $\pi(x)=\kappa\left(v_{1}, v_{2}, \ldots, v_{d}\right)$. An element $y$ is called a singleton element if it contains only one subtree. For an element $x_{i}=\left(\kappa\left(v_{i, 1}, v_{i, 2}, \ldots, v_{i, d}\right), T_{v_{i, 1}}, T_{v_{i, 2}}, \ldots, T_{v_{i, d}}\right)$ and a singleton element $y_{j}=\left(\kappa\left(v_{j}\right), T_{v_{j}}\right)$, if $T_{v_{j} \not \subset T_{v_{i, l}}}, 1 \leqslant l \leqslant d$, let $x_{i} \uplus y_{j}=\left(\kappa\left(v_{i, 1}, v_{i, 2}, \ldots, v_{i, d}, v_{j}\right), T_{v_{i, 1}}, T_{v_{i, 2}}, \ldots, T_{v_{i, d}}, T_{v_{j}}\right)$; if $T_{v_{j} \subset T_{v_{i}}}, 1 \leqslant l \leqslant d$, let $x_{i} \uplus y_{j}=(\kappa(\hat{V}), \hat{T})$, where the set $\hat{V}=\left\{v_{i, 1}, v_{i, 2}, \ldots, v_{i, d}, v_{j}\right\}-\left\{v_{i, l}\right\}$ and $\hat{T}=\left\{T_{v_{i, 1}}, T_{v_{i, 2}}, \ldots, T_{v_{i, d}}, T_{v_{j}}\right\}-\left\{T_{v_{i, l}}\right\}$. Let $h$ denote a half of the total weights of nodes in $C_{i}$. The Rooted-Tree Subset-Sum problem is to cut $C_{i}$ into minimal number of subtrees such that the total weights of nodes in the sub trees is equal to $h$. We formulate the Rooted-Tree Subset-Sum Problem as follows.

The Rooted-Tree Subset-Sum problem. Given a set $R$ of singleton elements associated with a rooted tree $C_{i}$ and an integer $h$, find an element $x$ derived by a sequence of $\uplus$ operations such that $\pi(x)=h$ and minimize $|x|$.

Theorem 1. The decision problem of the Rooted-Tree Subset-Sum problem is NP-complete. 
Proof. We first show that Rooted-Tree Subset-Sum problem is in NP. Given a set $R$ associated with a rooted tree and two integers $q$ and $h$, we let the subset $R^{\prime}$ of $R$ be the certificate. Checking whether $h=\pi\left(\uplus_{x \in R^{\prime}} x\right)$ and $\left|\uplus_{x \in R^{\prime}} x\right|=q$ can be accomplished by a verification algorithm in polynomial time.

The SUBSET-SUM problem is an NP-complete problem [11]. We now show that SUBSETSUM $\leqslant P$ Rooted-Tree Subset-Sum. Given an instance $\langle\hat{S}, t\rangle$ of the subset-sum problem, the reduction algorithm constructs a tree (a circuit) $C$ of the Rooted-Tree Subset-Sum problem such that there exists a subset in $\hat{S}$ whose sum is equal to $t$ if and only if there exists an element $x$ associated with $C$, where $\pi(x)=t$.

The heart of the reduction is a tree representation of $\hat{S}$. Let $\hat{S}=\left\{s_{1}, s_{2}, \ldots, s_{i}\right\}$ be a set consisting of $i$ integers. We construct the tree $C(V, N)$ with $i+1$ nodes associated with $\hat{S}$ as follows:

- Add a root $v_{0}$ with weight $\infty$ to $V$.

- For each integer $s_{j} \in S$, add a node $v_{j}$ with weight $s_{j}$ to $V$ and a directed edge $\left(v_{0}, v_{j}\right)$ to $N$.

Every subtree of $C$ except $T_{v_{0}}$ has only one node and is disjoint to each other. We have $R=\left\{\left(\kappa\left(v_{0}\right), T_{v_{0}}\right),\left(\kappa\left(v_{1}\right), T_{v_{1}}\right), \ldots,\left(\kappa\left(v_{i}\right), T_{v_{i}}\right)\right\}$ associated with $C(V, N)$. Let $q$ equal $i, \hat{S}^{\prime} \subseteq \hat{S}$ such that $t=\Sigma_{s_{j} \in \hat{S}^{\prime}} S_{j}$, and $y_{k}=\left(\kappa\left(v_{k}\right), T_{v_{k}}\right)$. Then we find the element $x=\uplus y_{j}$, where $y_{j}$ is associated with $s_{j} \in S^{i}$, such that $\pi(x)=t$ and $|x| \leqslant q$.

Conversely, suppose that there exists an element $x=\left(\kappa\left(v_{1}, v_{2}, \ldots, v_{d}\right), T_{v_{1}}, T_{v_{2}}, \ldots, T_{v_{d}}\right)$. Let $|x|$ equal $d$ and $\pi(x)$ equal $\kappa\left(v_{1}, v_{2}, \ldots, v_{d}\right)$ such that $\pi(x)=t$. Then, the sum of the subset $\left\{v_{j 1}, v_{j 2}, \ldots, v_{j k}\right\}$ is equal to $t$.

We give an exponential-time exact algorithm as well as a fully polynomial-time approximation scheme [11] for the Rooted-Tree Subset-Sum problem, listed in Figs. 6 and 7, respectively. For a sequence $L=<\left(\kappa\left(v_{1,1}, \ldots, v_{1, i_{1}}\right), T_{v_{1,1}}, \ldots, T_{v_{1, i_{1}}}\right), \quad\left(\kappa\left(v_{2,1}, \ldots, v_{2, i_{2}}\right), T_{v_{2,1}}, \ldots, T_{v_{2, i_{2}}}\right), \ldots$, $\left(\kappa\left(v_{m, 1}, \ldots, v_{m, i_{m}}\right), T_{v_{m, 1}}, \ldots, T_{v_{m, i_{m}}}\right)>$ and $\left(\kappa\left(v_{j}\right), T_{v_{j}}\right)$, let $L+\left(\kappa\left(v_{j}\right), T_{v_{j}}\right)$ denote the sequence derived from a series of $\uplus$ operations on each element of $L$ with the singleton element $\left(\kappa\left(v_{j}\right), T_{v_{j}}\right)$. For example, if $L=<\left(1, T_{v_{1}}\right),\left(3, T_{v_{2}}\right),\left(5, T_{v_{3}}\right),\left(6, T_{v_{4}}\right)>$, then $L+\left(2, T_{v_{5}}\right)=<\left(3, T_{v_{1}}, T_{v_{5}}\right)$, $\left(5, T_{v_{2}}, T_{v_{5}}\right),\left(7, T_{v_{3}}, T_{v_{5}}\right),\left(8, T_{v_{4}}, T_{v_{5}}\right)>$ (if $T_{v_{1}}, \ldots, T_{v_{5}}$ do not share any node).

We use an auxiliary procedure merge-lists $\left(L, L^{\prime}\right)$ that returns the sorted list by merging its two sorted input lists $L$ and $L^{\prime}$, and remove the duplicate elements. Like the merge procedure which used in merge sort [11], merge-lists runs in time $O\left(|L|+\left|L^{\prime}\right|\right)$. Since the length of $L_{i}$ can be as much as $2^{i}$, Exact-Rooted-Tree-Subset-Sum is an exponential-time algorithm.

The polynomial-time approximation algorithm Approx-Rooted-Tree-Subset-Sum is performed by trimming each list $L_{i}$ after an $\uplus$ operation. We use a trimming parameter $\varepsilon$ such that $0 \leqslant \varepsilon \leqslant 1$. To trim a list $L$ by $\varepsilon$ means to remove as many elements from $L$ as possible, in such a way that if $L^{\prime}$ is the result of trimming $L$, then for each element $y$ removed from $L$, there exists an element $z$ still in $L^{\prime}$, where $(1-\varepsilon) \pi(y) \leqslant \pi(z) \leqslant \pi(y)$. Line 3 initializes the list $L_{0}$ to be the list containing just the element $(0, \varnothing)$. Lines $4-5$ perform the $\uplus$ operation in a topological order. Lines 6 and 7 remove each element $x, \pi(x)>h$ and $|x|>q$. Line 8 performs trimming operations. We can show that Approx-Rooted-Tree-Subset-Sum listed in Fig. 7 runs in time polynomially in both $|R|$ and $1 / \varepsilon$; i.e., it is a fully polynomial-time approximation scheme [11]. 


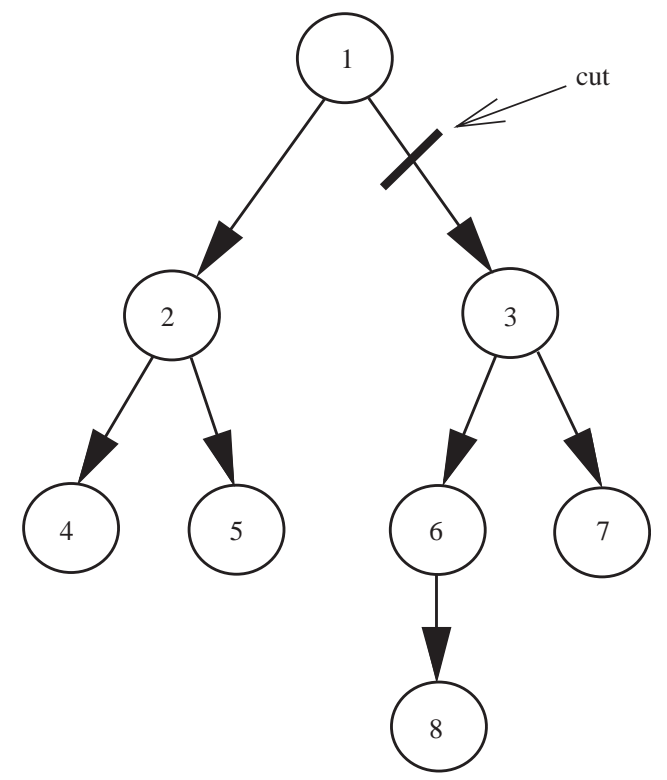

Fig. 5. A rooted-tree with eight vertices. The tree has a minimum cut (cut-size $=1$ ) which partitions the tree into two balanced parts.

We give an example of Approx-Rooted-Tree-Subset-Sum in the following. Suppose we have a list of singleton elements

$$
L=\left\langle\left(8, T_{v_{1}}\right),\left(3, T_{v_{2}}\right),\left(4, T_{v_{3}}\right),\left(1, T_{v_{4}}\right),\left(1, T_{v_{5}}\right),\left(2, T_{v_{6}}\right),\left(1, T_{v_{7}}\right),\left(1, T_{v_{8}}\right)\right\rangle
$$

associated with the rooted-tree in Fig. 5, in which the weight of each vertex is equal to 1 . The target is to find an element $x$, where $\pi(x)=h=4$ and $|x|=q=1$ with $\varepsilon=0.2$. The trimming parameter $\rho$ is $\varepsilon / 8=0.025$. The Approx-Rooted-Tree-Subset-Sum computes the elements as follows (Figs. $6 \&$ 7):

Line 2: $L_{0}=\langle(0, \emptyset)\rangle$,

Line 4: $\quad$ pick $\left(8, T_{v_{1}}\right)$,

Line 5: $L_{1}=\left\langle(0, \emptyset),\left(8, T_{v_{1}}\right)\right\rangle$,

Line 6: $L_{1}=\langle(0, \emptyset)\rangle$,

Line 7: $L_{1}=\langle(0, \emptyset)\rangle$,

Line 8: $L_{1}=\langle(0, \emptyset)\rangle$,

Line 4: $\quad$ pick $\left(3, T_{v_{2}}\right)$,

Line 5: $L_{2}=\left\langle(0, \emptyset),\left(3, T_{v_{2}}\right)\right\rangle$,

Line 6: $L_{2}=\left\langle(0, \emptyset),\left(3, T_{v_{2}}\right)\right\rangle$,

Line 7: $L_{2}=\left\langle(0, \emptyset),\left(3, T_{v_{2}}\right)\right\rangle$,

Line 8: $L_{2}=\left\langle(0, \emptyset),\left(3, T_{v_{2}}\right)\right\rangle$, 
Algorithm: Exact-Rooted-Tree-Subset-Sum $(R, h, q)$

Input: $R$-a set associated with a rooted tree $C_{i}(V, N)$.

$h$ : a target integer.

$q$ : an upper bound.

Output: $x^{*}$-an element s.t. $\pi\left(x^{*}\right)$ is as large as possible but not large than $h$ and $\left|x^{*}\right| \leq q$.

1. $l \leftarrow|R|$;

2. $L_{0} \leftarrow<(0, \emptyset)>$;

3. for $i \leftarrow 1$ to $l$ do

4. Pick $\left(\kappa\left(v_{j}\right), T_{v_{j}}\right) \in R$ according to the topological order of node $v_{j}$ in $C$;

5. $L_{i} \leftarrow$ Merge-Lists $\left(L_{i-1}, L_{i-1}+\left(\kappa\left(v_{j}\right), T_{v_{j}}\right)\right)$;

6. Remove each element $\left(\kappa\left(v_{i, 1}, \ldots, v_{i, d}\right), T_{v_{i, 1}}, \ldots, T_{v_{i, d}}\right)$ from $L_{i}$ s.t. $\kappa\left(v_{i, 1}, \ldots, v_{i, d}\right)$ is greater than $h$;

7. Remove each element $\left(\kappa\left(v_{i, 1}, \ldots, v_{i, d}\right), T_{v_{i, 1}}, \ldots, T_{v_{i, d}}\right)$ from $L_{i}$ s.t. $\left|\left(\kappa\left(v_{i, 1}, \ldots, v_{i, d}\right), T_{v_{i, 1}}, \ldots, T_{v_{i, d}}\right)\right|$ is greater than $q$;

8. Output the element $x^{*}$ in $L_{l}$ s.t. $\pi\left(x^{*}\right)$ is the largest.

Fig. 6. The exact algorithm for the Rooted-Tree Subset-Sum problem.

Line 4: $\quad$ pick $\left(4, T_{v_{3}}\right)$,

Line 5: $L_{3}=\left\langle(0, \emptyset),\left(3, T_{v_{2}}\right),\left(4, T_{v_{3}}\right),\left(7, T_{v_{2}}, T_{v_{3}}\right)\right\rangle$,

Line 6: $L_{3}=\left\langle(0, \varnothing),\left(3, T_{v_{2}}\right),\left(4, T_{v_{3}}\right)\right\rangle$,

Line 7: $L_{3}=\left\langle(0, \varnothing),\left(3, T_{v_{2}}\right),\left(4, T_{v_{3}}\right)\right\rangle$,

Line 8: $L_{3}=\left\langle(0, \emptyset),\left(3, T_{v_{2}}\right),\left(4, T_{v_{3}}\right)\right\rangle$,

Line 4: $\quad$ pick $\left(1, T_{v_{4}}\right)$,

Line 5: $L_{4}=\left\langle(0, \emptyset),\left(1, T_{v_{4}}\right),\left(3, T_{v_{2}}\right),\left(4, T_{v_{3}}\right),\left(5, T_{v_{3}}, T_{v_{4}}\right)\right\rangle$,

Line 6: $L_{4}=\left\langle(0, \emptyset),\left(1, T_{v_{4}}\right),\left(3, T_{v_{2}}\right),\left(4, T_{v_{3}}\right)\right\rangle$,

Line 7: $L_{4}=\left\langle(0, \emptyset),\left(1, T_{v_{4}}\right),\left(3, T_{v_{2}}\right),\left(4, T_{v_{3}}\right)\right\rangle$,

Line 8: $L_{4}=\left\langle(0, \emptyset),\left(1, T_{v_{4}}\right),\left(3, T_{v_{2}}\right),\left(4, T_{v_{3}}\right)\right\rangle$,

Line 4: $\quad$ pick $\left(1, T_{v_{5}}\right)$,

Line 5: $L_{5}=\left\langle(0, \emptyset),\left(1, T_{v_{4}}\right),\left(1, T_{v_{5}}\right),\left(2, T_{v_{4}}, T_{v_{5}}\right),\left(3, T_{v_{2}}\right),\left(4, T_{v_{3}}\right),\left(5, T_{v_{3}}, T_{v_{3}}\right)\right\rangle$,

Line 6: $L_{5}=\left\langle(0, \emptyset),\left(1, T_{v_{4}}\right),\left(1, T_{v_{5}}\right),\left(2, T_{v_{4}}, T_{v_{5}}\right),\left(3, T_{v_{2}}\right),\left(4, T_{v_{3}}\right)\right\rangle$,

Line 7: $L_{5}=\left\langle(0, \emptyset),\left(1, T_{v_{4}}\right),\left(1, T_{v_{5}}\right),\left(3, T_{v_{2}}\right),\left(4, T_{v_{3}}\right)\right\rangle$,

Line 8: $L_{5}=\left\langle(0, \emptyset),\left(1, T_{v_{4}}\right),\left(1, T_{v_{5}}\right),\left(3, T_{v_{2}}\right),\left(4, T_{v_{3}}\right)\right\rangle$, 
Algorithm: Approx-Rooted-Tree-Subset-Sum $(R, h, q, \epsilon)$

Input: $R$-a set associated with a rooted tree $C_{i}(V, N)$.

$h$ : a target integer.

$q$ : an upper bound.

$\epsilon$ : a trimming parameter.

Output: $x^{*}$-an element s.t. $\pi\left(x^{*}\right)$ is as large as possible but not large than $h$ and $\left|x^{*}\right| \leq q$.

1. $l \leftarrow|R|$;

2. $L_{0} \leftarrow<(0, \emptyset)>$;

3. for $i \leftarrow 1$ to $l$ do

4. Pick $\left(\kappa\left(v_{j}\right), T_{v_{j}}\right) \in R$ according to the topological order of node $v_{j}$ in $C$;

5. $L_{i} \leftarrow$ Merge-Lists $\left(L_{i-1}, L_{i-1}+\left(\kappa\left(v_{j}\right), T_{v_{j}}\right)\right)$;

6. Remove each element $\left(\kappa\left(v_{i, 1}, \ldots, v_{i, d}\right), T_{v_{i, 1}}, \ldots, T_{v_{i, d}}\right)$ from $L_{i}$ s.t. $\kappa\left(v_{i, 1}, \ldots, v_{i, d}\right)$ is greater than $h$;

7. Remove each element $\left(\kappa\left(v_{i, 1}, \ldots, v_{i, d}\right), T_{v_{i, 1}}, \ldots, T_{v_{i, d}}\right)$ from $L_{i}$ s.t. $\left|\left(\kappa\left(v_{i, 1}, \ldots, v_{i, d}\right), T_{v_{i, 1}}, \ldots, T_{v_{i, d}}\right)\right|$ is greater than $q$;

8. $L_{i} \leftarrow \operatorname{Trim}\left(L_{i}, \epsilon / l\right)$;

9. Output the element $x^{*}$ in $L_{l}$ s.t. $\pi\left(x^{*}\right)$ is the largest.

Subroutine: $\operatorname{Trim}\left(L_{i}, \epsilon\right)$

1. $m \leftarrow\left|L_{i}\right|$;

2. $L^{\prime} \leftarrow<y_{1}>$, where $y_{1}$ is the first element in $L_{i}$;

3. last $\leftarrow \pi\left(y_{1}\right)$;

4. for $i \leftarrow 2$ to $m$ do

5. if last $<(1-\epsilon) \pi\left(y_{i}\right)$ then

6. $\quad$ append $y_{i}$ onto the end of $L^{\prime}$;

7. last $\leftarrow \pi\left(y_{i}\right)$;

8. Output $L^{\prime}$

Fig. 7. The fully polynomial-time approximation scheme 0 the Rooted-Tree Subset-Sum problem.

Line 4: $\quad$ pick $\left(2, T_{v_{6}}\right)$,

Line 5: $L_{6}=\left\langle(0, \emptyset),\left(1, T_{v_{4}}\right),\left(1, T_{v_{5}}\right),\left(2, T_{v_{6}}\right),\left(3, T_{v_{2}}\right),\left(3, T_{v_{4}}, T_{v_{6}}\right),\left(3, T_{v_{5}}, T_{v_{6}}\right),\left(4, T_{v_{3}}\right)\right.$, $\left.\left(5, T_{v_{2}}, T_{v_{6}}\right)\right\rangle$,

Line 6: $L_{6}=\left\langle(0, \emptyset),\left(1, T_{v_{4}}\right),\left(1, T_{v_{5}}\right),\left(2, T_{v_{6}}\right),\left(3, T_{v_{2}}\right),\left(3, T_{v_{4}}, T_{v_{6}}\right),\left(3, T_{v_{5}}, T_{v_{6}}\right),\left(4, T_{v_{3}}\right)\right\rangle$, Line 7: $L_{6}=\left\langle(0, \emptyset),\left(1, T_{v_{4}}\right),\left(1, T_{v_{5}}\right),\left(2, T_{v_{6}}\right),\left(3, T_{v_{2}}\right),\left(4, T_{v_{3}}\right)\right\rangle$, Line 8: $L_{6}=\left\langle(0, \emptyset),\left(1, T_{v_{4}}\right),\left(1, T_{v_{5}}\right),\left(2, T_{v_{6}}\right),\left(3, T_{v_{2}}\right),\left(4, T_{v_{3}}\right)\right\rangle$, 
Line 4: $\quad \operatorname{pick}\left(1, T_{v_{7}}\right)$,

Line 5: $L_{7}=\left\langle(0, \emptyset),\left(1, T_{v_{4}}\right),\left(1, T_{v_{5}}\right),\left(1, T_{v_{7}}\right),\left(2, T_{v_{6}}\right),\left(2, T_{v_{4}}, T_{v_{7}}\right),\left(2, T_{v_{5}}, T_{v_{7}}\right),\left(3, T_{v_{2}}\right)\right.$, $\left.\left(3, T_{v_{6}}, T_{v_{7}}\right),\left(4, T_{v_{3}}\right),\left(4, T_{v_{2}}, T_{v_{7}}\right)\right\rangle$,

Line 6: $L_{7}=\left\langle(0, \emptyset),\left(1, T_{v_{4}}\right),\left(1, T_{v_{5}}\right),\left(1, T_{v_{7}}\right),\left(2, T_{v_{6}}\right),\left(2, T_{v_{4}}, T_{v_{7}}\right),\left(2, T_{v_{5}}, T_{v_{7}}\right),\left(3, T_{v_{2}}\right)\right.$, $\left.\left(3, T_{v_{6}}, T_{v_{7}}\right),\left(4, T_{v_{3}}\right),\left(4, T_{v_{2}}, T_{v_{7}}\right)\right\rangle$,

Line 7: $L_{7}=\left\langle(0, \emptyset),\left(1, T_{v_{4}}\right),\left(1, T_{v_{5}}\right),\left(1, T_{v_{7}}\right),\left(2, T_{v_{6}}\right),\left(3, T_{v_{2}}\right),\left(4, T_{v_{3}}\right),\left(4, T_{v_{2}}, T_{v_{7}}\right)\right\rangle$,

Line 8: $L_{7}=\left\langle(0, \emptyset),\left(1, T_{v_{4}}\right),\left(1, T_{v_{5}}\right),\left(1, T_{v_{7}}\right),\left(2, T_{v_{6}}\right),\left(3, T_{v_{2}}\right),\left(4, T_{v_{3}}\right)\right\rangle$,

Line 4: $\quad$ pick $\left(1, T_{v_{8}}\right)$,

Line 5: $L_{8}=\left\langle(0, \emptyset),\left(1, T_{v_{4}}\right),\left(1, T_{v_{5}}\right),\left(1, T_{v_{7}}\right),\left(1, T_{v_{8}}\right),\left(2, T_{v_{6}}\right),\left(2, T_{v_{4}}, T_{v_{8}}\right),\left(2, T_{v_{5}}, T_{v_{8}}\right)\right.$, $\left.\left(2, T_{v_{7}}, T_{v_{8}}\right),\left(3, T_{v_{2}}\right),\left(4, T_{v_{3}}\right),\left(4, T_{v_{2}}, T_{v_{8}}\right)\right\rangle$,

Line 6: $L_{8}=\left\langle(0, \emptyset),\left(1, T_{v_{4}}\right),\left(1, T_{v_{5}}\right),\left(1, T_{v_{7}}\right),\left(1, T_{v_{8}}\right),\left(2, T_{v_{6}}\right),\left(2, T_{v_{4}}, T_{v_{8}}\right),\left(2, T_{v_{5}}, T_{v_{8}}\right)\right.$, $\left.\left(2, T_{v_{7}}, T_{v_{8}}\right),\left(3, T_{v_{2}}\right),\left(4, T_{v_{3}}\right),\left(4, T_{v_{2}}, T_{v_{8}}\right)\right\rangle$

Line 7: $L_{8}=\left\langle(0, \emptyset),\left(1, T_{v_{4}}\right),\left(1, T_{v_{5}}\right),\left(1, T_{v_{7}}\right),\left(1, T_{v_{8}}\right),\left(2, T_{v_{6}}\right),\left(3, T_{v_{2}}\right),\left(4, T_{v_{3}}\right)\right.$, $\left.\left(4, T_{v_{2}}, T_{v_{8}}\right)\right\rangle$,

Line 8: $L_{8}=\left\langle(0, \emptyset),\left(1, T_{v_{4}}\right),\left(1, T_{v_{5}}\right),\left(1, T_{v_{7}}\right),\left(1, T_{v_{8}}\right),\left(2, T_{v_{6}}\right),\left(3, T_{v_{2}}\right),\left(4, T_{v_{3}}\right)\right\rangle$.

The algorithm returns $\left(4, T_{v_{3}}\right)$, where $\pi\left(4, T_{v_{3}}\right)=4$, which is bounded in $\varepsilon=20 \%$ of the optimal answer.

Theorem 2. Approx-Rooted-Tree-Subset-Sum is a fully polynomial-time approximation scheme for the Rooted-Tree Subset-Sum Problem.

Proof. In lines 6-8, the operation trimming $L_{i}$ and removes each element $y$ where $\pi(y)$ is greater than $h$ from $L_{i}$. The rest elements of $L_{i}$ are generated by selecting a subset of $R$ and applying a sequence of $\uplus$ operations on the selected elements. Therefore, the element $x^{*}$ returned in line 9 is indeed derived from a subset of $R$. It remains to show that the $\pi\left(x^{*}\right)$ is not smaller than $1-\varepsilon$ times an optimal solution, and we must also show that the algorithm runs in polynomial time.

To show that the relative error of the returned answer is small, note that when list $L_{i}$ is trimmed, we introduce a relative error of at most $\varepsilon / l$ between the representative $\pi$ values of the elements remaining and the $\pi$ values of the elements before trimming. By induction on $i$, it can be shown that for each possible element $y$ in $L_{i}$ produced by the Exact-Rooted-Tree-Subset-Sum algorithm, there exists an element $x \in L_{i}$ produced by the Approx-Rooted-Tree-Subset-Sum algorithm such that

$$
(1-\varepsilon / l)^{i} \pi(y) \leqslant \pi(x) \leqslant \pi(y) .
$$

If $y^{*}$ denotes an optimal solution to the Rooted-Tree Subset-Sum problem, then there is a $x^{*} \in L_{l}$ such that

$$
(1-\varepsilon / l)^{l} \pi\left(y^{*}\right) \leqslant \pi\left(x^{*}\right) \leqslant \pi\left(y^{*}\right)
$$


where $\pi\left(x^{*}\right)$ is the $\pi$ value of the element $x^{*}$ returned by Approx-Rooted-Tree-Subset-Sum. Since $l \geqslant 1>\varepsilon$, it can be shown that

$$
\frac{\mathrm{d}}{\mathrm{d} l}\left(1-\frac{\varepsilon}{l}\right)^{l}>0
$$

It implies that the function $(1-\varepsilon / l)^{l}$ increases with $l$, so that $l>1$ implies

$$
1-\varepsilon<(1-\varepsilon / l)^{l}
$$

and thus,

$$
(1-\varepsilon) \pi\left(y^{*}\right) \leqslant \pi\left(x^{*}\right) .
$$

Therefore, the $\pi$ value of $x^{*}$ returned by Approx-Rooted-Tree-Subset-Sum is not smaller than $1-\varepsilon$ times the $\pi$ value of the optimal solution $y^{*}$.

To show that this is a fully polynomial-time approximation scheme, we derive a bound on the length of $L_{i}$. After trimming, successive elements $x$ and $x^{\prime}$ of $L_{i}$ must have the relationship $\pi(x) / \pi\left(x^{\prime}\right)>1 /(1-\varepsilon / l)$. That is, their $\pi$ values must differ by a factor of at least $(1-\varepsilon / l)$. Therefore, the number of elements in each $L_{i}$ is at most

$$
\log _{1 /(1-\varepsilon / l)} h=\frac{\ln h}{-\ln (1-\varepsilon / l)} \leqslant \frac{l \ln h}{\varepsilon}
$$

since $\ln (1+i) \leqslant i$ for $i>-1$. This bound is polynomial in the number $l$ of the given input elements, in the number of bits $\ln h$ needed to represent $h$, and in $1 / \varepsilon$. Since the running time of Approx-Rooted-Tree-Subset-Sum is polynomial in the length of $L_{i}$, Approx-Rooted-Tree-SubsetSum is a fully polynomial-time approximation scheme.

Approx-Rooted-Tree-Subset-Sum tells us how to partition a rooted-tree circuit. If its results contain infeasible trees, we need to apply Approx-Rooted-Tree-Subset-Sum repeatedly.

For the case where $C_{i}$ (rooted at $v$ ) is an acyclic graph. We can perform breadth-first search from node $v$ and obtain a rooted tree, and then apply Approx-Rooted-Tree-Subset-Sum on the tree.

\subsection{Phase II: the probability-based algorithm}

The probability-based iterative-improvement method extends the work [12] to fit the architecture of Xilinx TMFPGAs.

\subsubsection{Iterative-improvement approach}

In the TMFPGA bipartitioning problem, the set $V$ of nodes is divided into two subsets $V_{1}$ and $V_{2}$, which represent nodes in two stages. For any two nodes $u, v$ in $V$, if $\operatorname{Pre}(u) \operatorname{Pre}(v)$, then $u, v$ are in the same stage, or $u$ is $V_{1}$ and $v$ is in $V_{2}$. Further, $V_{1}$ and $V_{2}$ must satisfy the balance constraint. The size of $\operatorname{Cut}(2,1)$ equals the number of total registers in the circuit, which cannot be reduced any more. Therefore, we only need to minimize the size of $\operatorname{Cut}(1,2)$ in the TMFPGA 
bipartitioning problem. In the second step of CPAT, we present the PAT (Probability-based Algorithm for TMFPGA), which applies a probability-based, iterative-improvement approach to minimize the size of $\operatorname{Cut}(1,2)$. (Fig. 10 summarizes PAT.) We first use the topological sort to obtain an initial partitioning that satisfies the balance and the precedence constraints (line 1 in Fig. 10). During the iterative improvement, each node is assigned a gain, representing the benefit of moving the node to the other subset. In each pass (lines 4-18 in Fig. 10), we choose a node with the largest gain and check if it will violate the balance or the precedence constraint after moving the node. If it is feasible to move the node, it is temporarily moved and locked. Select the best sequence of moves and make them permanent. Repeat the above process in a pass until no better cutsize is found.

\subsubsection{The precedence constraint}

Because of the precedence constraint, moving a node to the other subset may not be valid. For C-type nodes, we use the following two rules to judge if a node can be moved:

R1: A C-type node $v$ in $V_{1}$ can be moved if all its successors in $V_{1}$ have been moved.

R2: A C-type node $v$ in $V_{2}$ can be moved if all its ancestors in $V_{2}$ have been moved.

For example, in Fig. 8(a), $v_{2}$ cannot be moved according to Rule R1. In Fig. 8(b), $v_{3}$ cannot be moved according to Rule R2.

For FF-type nodes, we use the following rules to judge if a node can be moved:

R3: A FF-type node $v$ in $V_{2}$ can be moved if all its successors and ancestors in $V_{2}$ have been moved.

After a node $v$ is moved to the other stage, some of its neighbors may also be blocked in that stage due to the precedence constraint. We use the following two rules to determine whether such neighbors should be blocked (see line 14 in Fig. 10):

R4: If $v$ is moved from $V_{1}$ to $V_{2}$, all its successors should be blocked in $V_{2}$.

R5: If $v$ is moved from $V_{2}$ to $V_{1}$, all its ancestors should be blocked in $V_{1}$.

\subsubsection{Gains of nodes}

In the PAT, each node is given a probability for moving it to the other set. Based on these probabilities, an expected gain of moving a node to the other subset can be evaluated. Before detailing how to compute gains, we shall introduce some notation first.

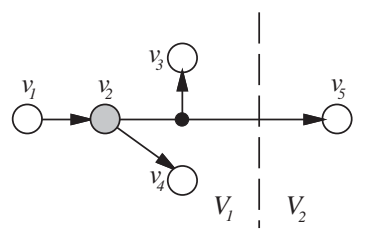

(a)

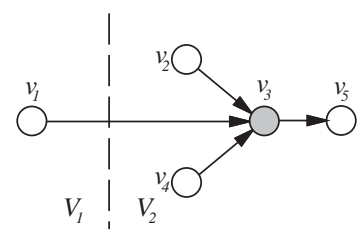

(b)

Fig. 8. The precedence constraints. Shaded nodes cannot be moved to the other stage due to the precedence constraints. 


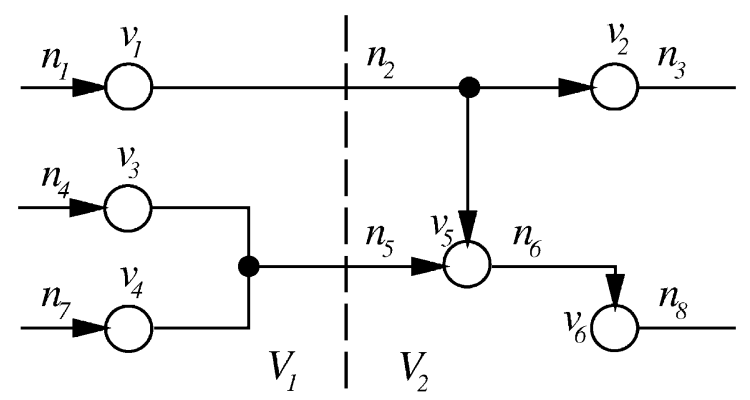

Fig. 9. Example for the notation.

- Cutset: set of nets which need micro registers in $\operatorname{Cut}(1,2)$. In other words, a C-type net is not in Cutset if all its nodes are in $V_{1}$ or $V_{2}$; an FF-type net is not in Cutset only if its fan-out node is in $V_{2}$ and all its fan-in nodes are in $V_{1}$.

- $c(n)$ : cost of net $n$.

- $p(u)$ : probability of moving node $u$ to the other stage.

- $n_{i}^{1 \rightarrow 2}$ : event that net $n_{i}$ is removed from Cutset by moving nodes to $V_{2}$. For a C-type net $n_{i}$ in Cutset, $n_{i}^{1 \rightarrow 2}$ means all its nodes in $V_{1}$ are moved to $V_{2}$; for an FF-type net $n_{i}$ in Cutset, $n_{i}^{1 \rightarrow 2}$ means all nodes are originally in $V_{1}$ and then its fan-out node is moved to $V_{2}$.

- $n_{i}^{2 \rightarrow 1}$ : event that net $n_{i}$ is removed from Cutset by moving nodes to $V_{1}$. For a C-type net $n_{i}$ in Cutset, $n_{i}^{2 \rightarrow 1}$ means all its nodes in $V_{2}$ are moved to $V_{1}$; for an FF-type net $n_{i}$ in Cutset, $n_{i}^{2 \rightarrow 1}$ means its fan-out node is originally in $V_{2}$ and then all its fan-in nodes are moved to $V_{1}$.

- $p\left(n_{i}^{a \rightarrow b}\right)$ : probability of net $n_{i}$ being removed from Cutset by moving all $n_{i}$ 's nodes in $V_{a}$ to $V_{b}$.

- $p\left(n_{i}^{a \rightarrow b} \mid u\right)$ : probability of removing net $n_{i}$ from Cutset by moving nodes to $V_{b}$ in the condition that node $u$ is originally in $V_{a}$ and then is moved to $V_{b}$.

- $p\left(n_{i}^{a \rightarrow b} \mid u^{c}\right)$ : probability of removing net $n_{i}$ from Cutset by moving nodes to $V_{b}$ in the condition that node $u$ is originally in $V_{b}$ and then stays in $V_{b}$.

- $f_{n_{i}}$ : fan-out node of net $n_{i}$.

- $S_{a}(u)$ : set of successors of $u$ in stage $V_{a}$.

- $A_{a}(u)$ : set of ancestors of $u$ in stage $V_{a}$.

- $E_{a}\left(n_{i}, n_{j}\right)$ : set of nodes in $V_{a}$ that are both in nets $n_{i}$ and $n_{j}$. E.g., in Fig. $9, E_{1}\left(n_{5}, n_{6}\right)=\emptyset$ and $E_{2}\left(n_{5}, n_{6}\right)=\left\{v_{5}\right\}$.

- $N_{a}(u)$ : set of $u$ 's neighbors in $V_{a}$.

- $I(u)$ : set of nets which contain node $u$.

- $M_{a}(n)$ : set of nets that have common nodes with net $n$ in $V_{a}$. E.g., in Fig. 9, $M_{1}\left(n_{5}\right)=\left\{n_{4}, n_{7}\right\}$ and $M_{2}\left(n_{5}\right)=\left\{n_{2}, n_{6}\right\}$.

- $e\left(n_{i}^{a \rightarrow b}\right)$ : expected gain in the condition that net $n_{i}$ is moved from $V_{a}$ to $V_{b}$.

- $e_{n_{j}}\left(n_{i}^{a \rightarrow b}\right)$ : expected gain contributed by $n_{j}$ in the condition that net $n_{i}$ is moved from $V_{a}$ to $V_{b}$.

- $g(u)$ : gain of node $u$.

- $g_{n_{j}}(u)$ : gain of node $u$ contributed by $n_{j}$. 
According to the definitions of $n_{i}^{1 \rightarrow 2}$ and $n_{i}^{2 \rightarrow 1}$, we have the following equations. For a C-type net $n_{i}$ with node $u, u \in V_{a}$,

$$
\begin{aligned}
& p\left(n_{i}^{a \rightarrow b} \mid u\right)=\prod_{v \in N_{a}\left(n_{i}\right)-\{u\}} p(v) \\
& p\left(n_{i}^{b \rightarrow a} \mid u^{c}\right)=\prod_{v \in N_{b}\left(n_{i}\right)} p(v) .
\end{aligned}
$$

For an FF-type net $n_{i}$ with node $u, \forall v, v \in V_{1}$ and $v \in n_{i}$,

$$
\begin{aligned}
& p\left(n_{i}^{1 \rightarrow 2} \mid u\right)= \begin{cases}1 & \text { if } u=f_{n_{i}} \\
0 & \text { otherwise }\end{cases} \\
& p\left(n_{i}^{1 \rightarrow 2} \mid u^{c}\right)= \begin{cases}p\left(f_{n_{i}}\right) & \text { if } u \neq f_{n_{i}} \\
0 & \text { otherwise }\end{cases} \\
& p\left(n_{i}^{2 \rightarrow 1} \mid u\right)=0 .
\end{aligned}
$$

For an FF-type net $n_{i}$ with node $u, f_{n_{i}} \in V_{2}$,

$$
\begin{aligned}
& p\left(n_{i}^{2 \rightarrow 1} \mid u\right)=\left\{\begin{array}{lc}
\prod_{v \in N_{2}\left(n_{i}\right)-\left\{f_{n_{i}}, u\right\}} p(v) & \text { if } u \in N_{2}\left(n_{i}\right)-\left\{f_{n_{i}}\right\} \\
0 & \text { otherwise }
\end{array}\right. \\
& p\left(n_{i}^{2 \rightarrow 1} \mid u^{c}\right)=\left\{\begin{array}{lc}
\prod_{v \in N_{2}\left(n_{i}\right)-\left\{f_{n_{i}}\right\}} p(v) & \text { if } u \in N_{1}\left(n_{i}\right) \\
0 & \text { otherwise }
\end{array}\right. \\
& p\left(n_{i}^{1 \rightarrow 2} \mid u\right)=0 .
\end{aligned}
$$

Moving a net $n_{i}$ to some stage will affect the move of the other nets that have common nodes with net $n_{i}$. It is called the 2 nd-order information [12]. Therefore, the expected gain for removing a net from Cutset should be considered.

$$
e\left(n_{i}^{a \rightarrow b}\right)=\sum_{n_{j} \in M_{a}\left(n_{i}\right)} e_{n_{j}}\left(n_{i}^{a \rightarrow b}\right) .
$$

For two C-type nets $n_{i}$ and $n_{j}, n_{i} \cap n_{j} \neq \emptyset$,

$$
e_{n_{j}}\left(n_{i}^{a \rightarrow b}\right)=c\left(n_{j}\right) p\left(n_{j}^{a \rightarrow b}\right) / \prod_{v \in E_{a}\left(n_{i}, n_{j}\right)} p(v) .
$$

For a C-type net $n_{i}$ and an FF-type net $n_{j}, n_{i} \cap n_{j} \neq \emptyset$,

(1) if $f_{n_{j}} \in V_{1}$,

$$
\begin{aligned}
& e_{n_{j}}\left(n_{i}^{1 \rightarrow 2}\right)=\left\{\begin{array}{lc}
c\left(n_{j}\right) & \text { if } E_{1}\left(n_{i}, n_{j}\right)=\left\{f_{n_{j}}\right\} \\
0 & \text { otherwise }
\end{array}\right. \\
& e_{n_{j}}\left(n_{i}^{2 \rightarrow 1}\right)=0
\end{aligned}
$$


(2) if $f_{n_{j}} \in V_{2}$,

$$
\begin{aligned}
& e_{n_{j}}\left(n_{i}^{2 \rightarrow 1}\right)=\left\{\begin{array}{lc}
\frac{c\left(n_{j}\right) p\left(n_{j}^{2 \rightarrow 1}\right)}{\prod_{v \in E_{2}\left(n_{i}, n_{j}\right)} p(v)} & \text { if } f_{n_{j}} \notin E_{2}\left(n_{i}, n_{j}\right) \\
0 & \text { otherwise }
\end{array}\right. \\
& e_{n_{j}}\left(n_{i}^{1 \rightarrow 2}\right)=0 .
\end{aligned}
$$

For an FF-type net $n_{i}$ and a C-type net $n_{j}, n_{i} \cap n_{j} \neq \emptyset$,

$$
\begin{aligned}
& e_{n_{j}}\left(n_{i}^{1 \rightarrow 2}\right)= \begin{cases}c\left(n_{j}\right) p\left(n_{j}^{1 \rightarrow 2}\right) / p\left(f_{n_{i}}\right) & \text { if } f_{n_{i}} \in n_{j} \\
0 & \text { otherwise }\end{cases} \\
& e_{n_{j}}\left(n_{i}^{2 \rightarrow 1}\right)= \begin{cases}\frac{c\left(n_{j}\right) p\left(n_{j}^{2 \rightarrow 1}\right)}{\prod_{v \in E_{2}\left(n_{i}, n_{j}\right)}^{p(v)}} & \text { if } f_{n_{i}} \notin n_{j} \\
0 & \text { otherwise. }\end{cases}
\end{aligned}
$$

For two FF-type nets $n_{i}$ and $n_{j}, n_{i} \cap n_{j} \neq \emptyset$,

(1) if $f_{n_{j}} \in V_{1}$,

$$
\begin{aligned}
& e_{n_{j}}\left(n_{i}^{1 \rightarrow 2}\right)= \begin{cases}c\left(n_{j}\right) & \text { if } f_{n_{i}}=f_{n_{j}} \\
0 & \text { otherwise }\end{cases} \\
& e_{n_{j}}\left(n_{i}^{2 \rightarrow 1}\right)=0 .
\end{aligned}
$$

(2) if $f_{n_{j}} \in V_{2}$,

$$
\begin{array}{ll}
e_{n_{j}}\left(n_{i}^{2 \rightarrow 1}\right)= \begin{cases}\frac{c\left(n_{j}\right) p\left(n_{j}^{2 \rightarrow 1}\right)}{\prod_{v \in E_{2}\left(n_{i}, n_{j}\right)-\left\langle f_{n_{i}}\right)^{\prime}} p(v)} & \text { if } f_{n_{j}} \notin n_{i} \\
0 & \text { otherwise }\end{cases} \\
e_{n_{j}}\left(n_{i}^{1 \rightarrow 2}\right)=0 .
\end{array}
$$

If net $n_{j}$ in the above cases is not in Cutset originally and moved into Cutset in condition of $n_{i}^{a \rightarrow b}$, the term $-c\left(n_{j}\right)$ should be incorporated into $e_{n_{j}}\left(n_{i}^{a \rightarrow b}\right)$. For example, two C-type nets $n_{i}$ and $n_{j}$, $n_{j} \in V_{a}$,

$$
e_{n_{j}}\left(n_{i}^{a \rightarrow b}\right)=-c\left(n_{j}\right)+c\left(n_{j}\right) p\left(n_{j}^{a \rightarrow b}\right) / \prod_{v \in E_{a}\left(n_{i}, n_{j}\right)} p(v) .
$$

Using the above equations, we can compute $g_{n_{i}}(u)$ as follows:

(1) if $n_{i}$ is C-type,

$$
g_{n_{i}}(u)=\left(c\left(n_{i}\right)+e\left(n_{i}^{a \rightarrow b}\right)\right) p\left(n_{i}^{a \rightarrow b} \mid u\right)-\left(c\left(n_{i}\right)+e\left(n_{i}^{b \rightarrow a}\right)\right) p\left(n_{i}^{b \rightarrow a} \mid u^{c}\right) .
$$

(2) if $n_{i}$ is FF-type,

$$
g_{n_{i}}(u)=\left(c\left(n_{i}\right)+e\left(n_{i}^{a \rightarrow b}\right)\right) p\left(n_{i}^{a \rightarrow b} \mid u\right)-\left(c\left(n_{i}\right)+e\left(n_{i}^{a \rightarrow b}\right)\right) p\left(n_{i}^{a \rightarrow b} \mid u^{c}\right) .
$$




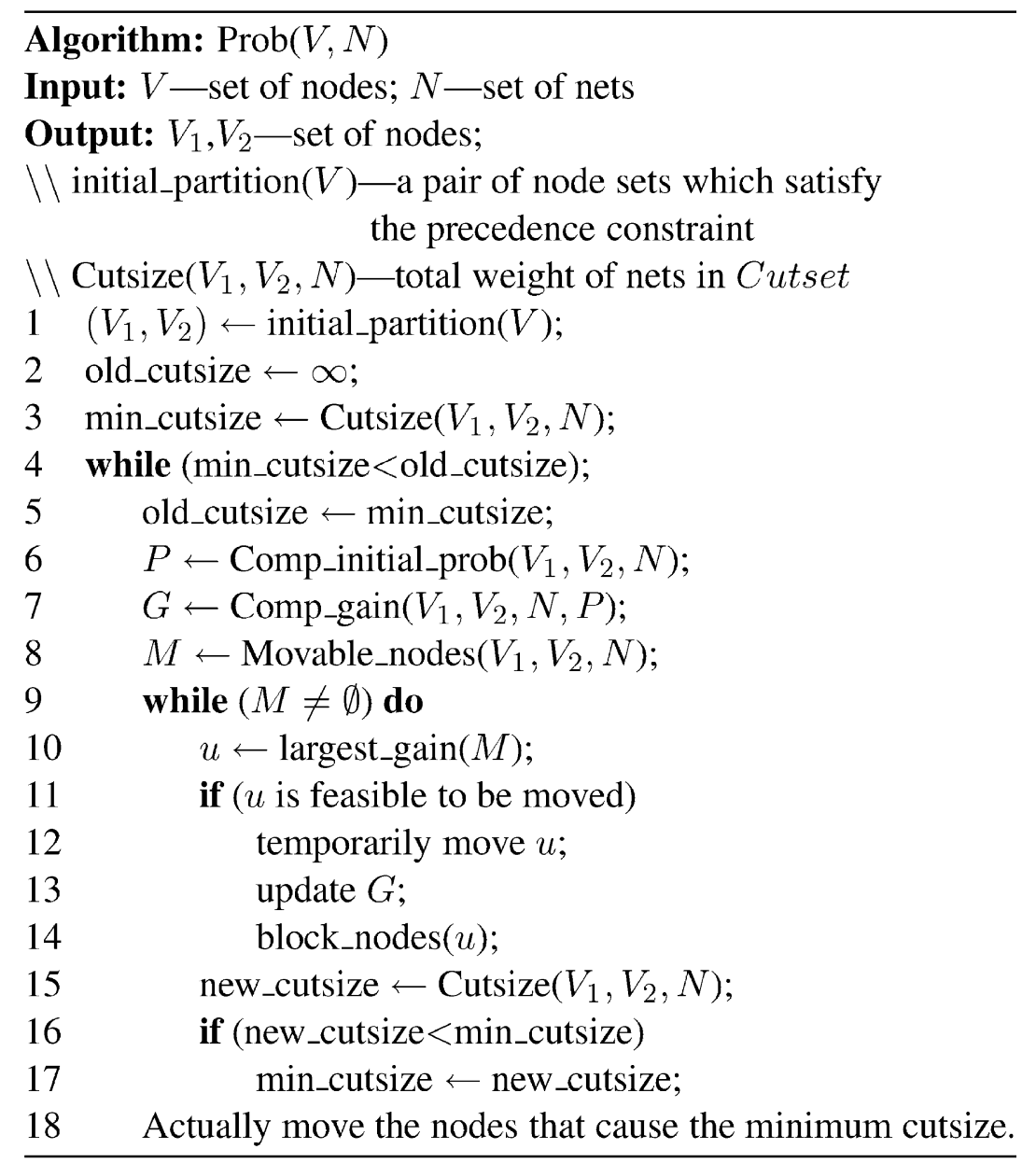

Fig. 10. The 2nd phase of CРAT: PAT.

Thus, the gain of a node $u$ is given by

$$
g(u)=\sum_{n_{i} \in I(u)} g_{n_{i}}(u)
$$

The probability of a node represents the likelihood that the node will be moved. The node with a greater gain has a higher probability to be moved. Thus, we can get the probability of a node by a monotonically increasing mapping function of its gain. (In our experiments shown in the next section, we used an increasing linear function.) It causes an interdependency between probabilities and gains since we obtain the gains from probabilities of nodes as shown in the above equations. To break this endless recursive relation, we give each node the probability 0.5 in our experiment. Repeat computing gains and probabilities from each other until they are stable enough, and then we have initial probabilities (line 6 in Fig. 10). In practice, three iterations are enough to reach a stable state. The probability-based algorithm PAT is summarized in Fig. 10. 


\subsection{The timing constraint}

The speed of a TMFPGA is determined by the maximum execution time of a micro-cycle. Therefore, we must reduce the longest path in a micro-cycle. In PAT, the lengths of the longest paths in both stages are upper bounded by $\lceil D / 2\rceil$, where $D$ is the length of the longest path in the circuit.

For a node $v$, let $\delta_{O}(v)$ denote the length of the longest path from $v$ to primary outputs and $\delta_{I}(v)$ denote the length of the longest path from primary inputs to $v$. A node $v$ cannot be put in $V_{1}$ if $\delta_{I}(v)$ is more than $\lceil D / 2\rceil$, because there will exist a path of length more than $\lceil D / 2\rceil$ from a primary input to $v$ in $V_{1}$. For the same reason, a node $v$ cannot be put in $V_{2}$ if $\delta_{O}(v)$ is more than $\lceil D / 2\rceil$. According to the above rules, the nodes that may violate the timing constraint are fixed in proper stages before the clustering phase.

\section{Experimental results}

The probability-based algorithm, $\mathrm{PAT}$, and the clustering- and probability-based algorithm, CPAT, were implemented in the $\mathrm{C}++$ language on a $\mathrm{PC}$ with a Pentium II 300 microprocessor and $128 \mathrm{MB}$ RAM and tested on the MCNC Partitioning93 benchmark circuits. The characteristics of the circuits are shown in Table 1. Columns 2 and 3 in Table 1 list the numbers of nodes and nets, respectively, in each circuit. In Table 2, we compare PAT and CPAT. Columns 2, 3, and 4 in Table 2 compare the maximum numbers of micro registers. Columns 5-7 compare the runtimes. The results show that PAT has performance for smaller circuits (e.g. the circuits size are less than 6000 in the benchmark circuits) while CPAT obtain better results for larger circuits (e.g. the circuits size are larger than 6000 in the benchmark circuits). It implies that the clustering algorithm in CPAT leads to a considerable improvement as the size of a circuit increases over a certain bound. In addition, the clustering algorithm in CPAT substantially reduces the problem size and thus the runtime. However, the clustering algorithm bound some nodes into a cluster. In the following probability-based algorithm, all nodes must be considered moving or not together in a cluster. Therefore, the clustering algorithm in CPAT might break the connectivities of nodes and nets when the circuit is small, in which the following probability-based algorithm might not be able to get the sufficient information to find a better result.

Table 1

Benchmark circuit characteristics

\begin{tabular}{lllllr}
\hline Circuit & No. of Nodes & No. of Nets & Circuit & No. of Nodes & No. of Nets \\
\hline c3540 & 1038 & 1016 & s9234 & 6098 & 5846 \\
c5315 & 1778 & 1655 & s13207 & 9445 & 8653 \\
c6288 & 2856 & 2824 & $\mathrm{~s} 15850$ & 11071 & 10385 \\
c7552 & 2247 & 2140 & $\mathrm{~s} 35932$ & 19880 & 17830 \\
s820 & 340 & 314 & $\mathrm{~s} 38417$ & 25589 & 23845 \\
s838 & 495 & 459 & $\mathrm{~s} 38584$ & 22451 & 20719 \\
s1423 & 831 & 750 & & & \\
\hline
\end{tabular}


Table 2

Comparison between PAT and CPAT

\begin{tabular}{|c|c|c|c|c|c|c|}
\hline \multirow[t]{2}{*}{ Circuit } & \multicolumn{3}{|c|}{ Max No. of registers } & \multicolumn{3}{|c|}{ Runtime (s) } \\
\hline & PAT & CPAT & Imprv. $(\%)$ & PAT & CPAT & Imprv. $(\%)$ \\
\hline c3540 & 126 & 152 & -17.1 & 3 & 3 & 0 \\
\hline c5315 & 157 & 174 & -9.8 & 11 & 4 & +63.7 \\
\hline s820 & 43 & 61 & -29.5 & 4 & 2 & +50.0 \\
\hline s838 & 72 & 93 & -22.6 & 1 & 1 & 0 \\
\hline s1423 & 106 & 120 & -11.7 & 3 & 2 & +33.3 \\
\hline s9234 & 430 & 402 & +6.5 & 29 & 25 & +13.8 \\
\hline s13 207 & 838 & 838 & 0 & 190 & 136 & +28.4 \\
\hline s15850 & 808 & 767 & +5.0 & 163 & 104 & +36.2 \\
\hline s35932 & 2138 & 2018 & +5.6 & 20131 & 15715 & +21.9 \\
\hline s38 417 & 2628 & 2468 & +6.0 & 1125 & 926 & +17.7 \\
\hline s38 584 & 3611 & 1451 & +59.8 & 1766 & 932 & +47.2 \\
\hline Average & & & -0.7 & & & +28.4 \\
\hline
\end{tabular}

Table 3

Results for the 8-stages TMFPGA partitioning of the smaller circuits

\begin{tabular}{|c|c|c|c|c|c|c|c|}
\hline \multirow[t]{2}{*}{ Circuit } & \multicolumn{4}{|c|}{ Max No. of registers } & \multicolumn{3}{|c|}{ PAT Imprv. (\%) } \\
\hline & List & FBP-m & Ref. [7] & PAT & List & FBP-m & Ref. [7] \\
\hline c3540 & 177 & 166 & 198 & 126 & +28.8 & +24.0 & +33.4 \\
\hline c5315 & 265 & 165 & 140 & 157 & +40.7 & +5.1 & -12.1 \\
\hline c6288 & 117 & 114 & 83 & 114 & +2.6 & 0 & -37.3 \\
\hline c7552 & 453 & 392 & 210 & 260 & +42.6 & +33.7 & -23.8 \\
\hline s820 & 91 & 81 & 52 & 43 & +52.7 & +46.9 & +17.3 \\
\hline s838 & 131 & 71 & 70 & 72 & +64.8 & -1.4 & -2.9 \\
\hline s1423 & 130 & 120 & 101 & 106 & +18.5 & +11.7 & -5.0 \\
\hline Average & & & & & +35.8 & +15.5 & +1.0 \\
\hline
\end{tabular}

In Table 3 (Table 4), we compared the performance of the smaller (larger) circuits of PAT (CPAT) with the approach in [7], the network-flow-based approach FBP-m [5], and the list scheduling List $[3,4]$ on the Xilinx TMFPGA model, in which a circuit was partitioned into eight stages. The size of a stage is bounded by the balance factor $5 \%$ (the same as in [5]). Columns $2-5$ list the maximum numbers of micro registers used by List, FBP-m, [7], and PAT, respectively. Columns 6-8 list the percentages of improvements of PAT over List, FBP-m, and [7], respectively. The results show that our PAT and CPAT algorithms outperform List and FBP-m by respective average reductions of $35.8 \%(40.0 \%)$ and $15.5 \%(22.3 \%)$ in the maximum numbers 
Table 4

Number of registers needed for CPAT and the previous works of the larger circuits

\begin{tabular}{|c|c|c|c|c|c|c|c|}
\hline \multirow[t]{2}{*}{ Circuit } & \multicolumn{4}{|c|}{ Max No. of registers } & \multicolumn{3}{|c|}{ PAT Imprv. (\%) } \\
\hline & List & FBP-m & Ref. [7] & CPAT & List & FBP-m & Ref. [7] \\
\hline s9234 & 640 & 502 & 381 & 402 & +37.2 & +19.9 & -5.5 \\
\hline s13207 & 1118 & 901 & 688 & 838 & +25.0 & +7.0 & -21.8 \\
\hline $\mathrm{s} 15850$ & 1070 & 877 & 761 & 767 & +28.3 & +12.5 & -0.8 \\
\hline s35932 & 3806 & 2950 & 2729 & 2018 & +47.0 & +31.6 & +26.1 \\
\hline s38 417 & 3546 & 2892 & 2194 & 2468 & +30.4 & +14.7 & -12.5 \\
\hline s38 584 & 5131 & 2796 & 2280 & 1451 & +71.7 & +48.1 & +36.4 \\
\hline Average & & & & & +40.0 & +22.3 & +3.7 \\
\hline
\end{tabular}

of micro registers required, and comparable to the algorithm in [7]. It implies that the probabilitybased scheme is effective in reducing the interconnection for TMFPGAs.

\section{Conclusion}

We have presented the probability-based algorithm PAT for the TMFPGA partitioning problem. Experimental results have shown that our probability-based algorithm outperforms the previous works, the List scheduling and the network-flow-based method, by significant margins. Furthermore, we can further improve the results for large circuits and runtimes for all circuits by incorporating a clustering algorithm into PAT.

\section{Acknowledgements}

The authors would like to thank Dr. Huiqun Liu for providing the benchmark circuits and helpful discussions on [5] and Prof. Ting-Chi Wang for his constructive comments.

\section{References}

[1] S. Trimberger, A time-multiplexed FPGA, in: Proceedings of FCCM, 1997, pp. 22-28.

[2] N.B. Bhat, et al., Performance-oriented fully routable dynamic architecture for a field programmable logic device, Memorandum No. UCB/RELM93/42, UC Berkeley, 1993.

[3] D. Chang, M. Marek-Sadowska, Buffer minimization and Time-multiplexed I/O on dynamically reconfigurable FPGAs, in: Proceedings of the FPGA Symposium, 1997, pp. 142-148.

[4] D. Chang, M. Marek-Sadowska, Partitioning sequential circuits on dynamically reconfigurable FPGAs, IEEE Trans. Comput. (1999) 565-578.

[5] H. Liu, D.F. Wong, Network flow based circuit partitioning for time-multiplexed FPGAs, in: Proceedings of ICCAD, 1998, pp. 497-504. 
[6] H. Liu, D.F. Wong, A graph theoretic optimal algorithm for schedule compression in time-multiplexed FPGA partitioning, in: Proceedings of ICCAD, 1999, pp. 400-405.

[7] W.K. Mak, F.Y. Young, Temporal logic replication for dynamically reconfigurable FPGA partitioning, in: Proceedings of ISPD, 2002, pp. 190-195.

[8] C.M. Fidducia, R.M. Mattheyses, A linear-time heuristic for improving network partitions, in: Proceedings of DAC, 1982, pp. 175-181.

[9] B.W. Kernighan, S. Lin, An efficient heuristic procedure for partitioning graphs, Bell System Technol. J. 49 (1970) 291-307.

[10] J. Cong, et al., Large scale circuit partitioning with loose/stable net removal and signal flow based clustering, in: Proceedings of ICCAD, 1997, pp. 441-446.

[11] T.H. Cormen, C.E. Leiserson, R.L. Rivest, in: Introduction to Algorithms, The MIT Press, Cambridge, MA, 1990, pp. 951-983.

[12] S. Dutt, W. Deng, Partitioning using second-order information and stochastic-gain functions, in: Proceedings of International Symposium Physical Design, 1998, pp. 112-117. 